\title{
An immunological approach
}

Barry Bloom, professor of microbiology at New York's

Albert Einstein College of Medicine, looks at the possibilities for preventing parasitic diseases in cattle

$\mathrm{W}$

ITH projections that world population will increase from 4,000 million to about 7,500 million by the end of the century, and an estimated 500 million people currently suffering from malnutrition, the magnitude of the world food crisis has finally touched the consciousness of people everywhere. Attention has been focused primarily on the green revolution and the need for increasing production of food grains; consequently the need for increasing production of meat and cattle has for the most part been overlooked.

The present situation is grim. While reliable statistics are virtually nonexistent, millions of head of cattle die of parasitic diseases each year, and more than 700 million are estimated to be at risk. In Africa alone, at least 7 million square kilometres of grazeable land, capable of supporting 120 million head of cattle, remain largely unproductive, chiefly because of tryanosomiasis and East Coast fever. The use of pesticides, while clearly important, has been inadequate to stave off these diseases, which are carried by the tsetse fly and ticks respectively. Effective drugs either do not exist or are too expensive for general use.

As a consequence of the enormous losses in potential food resources, there has recently been some discussion of the possibility of developing vaccines for immunizing cattle against diseases caused by blood parasites. In particular, the Rockefeller Foundation has sponsored a series of meetings to discuss the prospects.

Advocates of an immunological approach to control of parasitic diseases draw encouragement from two simple observations. First, if there were no immunity to such diseases, there would probably be no cattle in most of sub-Saharan Africa. Second, although mortality from these diseases is distressingly high, animals which survive infection are often resistant to subsequent reinfection. In fact, animals native to Africa, such as the n'dama, appear to be more resistant to parasitic diseases than species introduced from Europe. The general hope is that, with better understanding of the immune mechanisms required for protection, better characterisation of the parasite antigens required for resistance, and the use of immunological adiuvantswhich are used remarkably infrequently in parasitic immunology-an immunological approach to control of these diseases could be realistic.
Although research has so far been limited, there have already been some promising results. Vaccines have been developed for protecting cattle against three parasitic diseases (babesiosis, anaplasmosis and East Coast fever) and immunisation has been found to offer protection against three others in laboratory animals (malaria, South American and African trypanosomiasis).

Even beyond our current wealth of ignorance of the specific aspects of host-parasite relationships, there exist serious problems which limit real progress in this area. A major problem is money. According to recent figures from the World Health Organisation, for example, in 25 African countries for which details are available, the average annual health budget is $\$ 1.17$ per capita, and in 8 countries it is less than \$1 per capita. In seven of those countries, the health budget is less than $5 \%$ of the total government budget. If those figures represent levels of support for control of human disease, the prospects for control of animal diseases by the developing countries themselves are clearly dismal. Moreover, the more developed countries hardly seem to be concerned with these problems-in recent years, there has been a general reduction in bilateral and international assistance programs in health-related areas.

A further problem is the increasing criticism of meat production and consumption in the West. While this campaign has relevance to grainintensive cattle production in the developed countries, there is a danger that it may inhibit development of important food resources in developing nations where grain-intensive feeding is neither necessary nor possible. In sub-Saharan Africa, for example, cattle feed on low grade brush and natural vegetation, on lands generally too poor to support cultivated crops.

\section{Promising developments}

In spite of those problems, however, there have been some promising developments against the following widespread parasitic diseases.

- The most enigmatic and biologically fascinating of the animal parasite diseases is East Coast fever, caused by Theileria parvum, a virtually unclassifiable tick-borne parasite. The disease, which takes the form of a galloping proliferation of lymphocytes, not unlike leukemia, is responsible for the death of half a million cattle a year.

\section{Sorry, for copyright reasons some images on this page may not be available online}

The extraordinary aspect of the disease in cattle is that the parasite grows only in lymphocytes, and appears to act in a manner similar to cancer-causing viruses in tranforming lymphocytes into continuously growing cell lines.

There has been progress in producing immunity in cattle in two ways: by infecting them with ticks, followed by antibiotic treatment, and more recently, by immunising them with infected lymphocyte cultures. The hope is that the parasite can be isolated from infected lymphocyte cultures, and the protective antigens purified and characterised.

- A million young cattle die each year from another tick-borne disease, anaplasmosis. It is caused by a family of rickettsiae which infect and multiply within mature erythrocytes. An attenuated vaccine has been produced by subjecting the parasite to gamma radiation and passing it repeatedly through an unnatural host, the sheep. Striking protection against tick infection has been observed in small scale experimental trials in the United States, and more extensive field trials are under way in Mexico and Columbia.

- A third tick-borne disease, babesiosis, is responsible for the death of about a quarter of a million cattle a year. The parasite is related to the malaria parasite, and similarly multiples in and destroys erythrocytes. An attenuated organism has been produced by passing the parasite through splenectomised cattle, and a highly effective vaccine, currently in use in Australia, has been developed

- African trypanosomiasis, the most serious of the animal diseases, is responsible for the death of 3 million cattle a year. The animal discase and human sleeping sickness, both of which are caused by parasites carried by the tsetse fly, are clinically very similar. They represent a very difficult immunological problem because the parasite 
has developed a highly effective method for eluding the immune system of the host, known as 'antigenic variation'. The disease is characterised by waves of illness, reflecting rapid growth of the parasite, followed by remission engendered by an immune response, followed by sequential emergence of antigenically different organisms.

Because the organism has not yet been grown in culture, it is not known by what genetic or regulatory mechanism it manages to change its surface characteristics to evade the immune system. Two different lines of research for potential vaccination are, however, being followed. The first would involve modifying the immune system of the host so that it would react quickly to wipe out the second appearance of the organism before it reaches sufficient numbers to produce disease. Following the recent isolation of the major antigenic surface molecule, the second would involve the production of a "cocktail" vaccine containing a mixture of antigens from the most common variants to combat the different forms of the organism. Both require better characterisation of the organism and its antigens.

- There are many similarities between the animal and human parasitic diseases, such as Chagas disease and malaria, and there are consequently common research problems and strat- egies. Chagas disease, caused by the organism Trypanosoma cruzi, affects 30 million people, chiefly in Latin America. Ten per cent of its victims, virtually all children, die from an acute disease, while the rest develop a chronic illness with invasion and destruction of heart and smooth muscle. It frequently ends in sudden death. Although there is no effective treatment at present, an irradiated vaccine has been produced from parasites grown in animal cell cultures. It provides virtually $100 \%$ protection to massive challenge in susceptible mice, which are killed by a single virulent trypanosome.

- Two hundred and fifty milion people now suffer from malaria worldwide, and at least a million people die from the disease in Africa alone. Two successful types of experimental vaccines against different forms of the organism have been produced, but practical limitations on the quantity of these vaccines preclude either of them from being useful on a large scale. One line of research is aimed at defining the nature of the protective antigens so that synthetic antigens could be developed as potential vaccine material.

There are major scientific questions to be understood and problems to be overcome that will require contributions by molecular biologists and geneticists as well as parasitologists and immunologists, and at this stage even if increased support for research were forthcoming, it would be foolish to guarantee that inexpensive and effective vaccines and drugs would result. Even if some were to be developed, there would remain two further serious problems. Although effective vaccines, particularly against African trypanosomiasis, would offer the possibility of opening vast areas in the tsetse belt to cattle production, without thoughtful assessment of the ecological consequences and intelligent regulation, marginal areas of the Sahel and tropical Africa could be destroyed by overgrazing. The second problem is the lack of an effective health infrastructure in the developing countries. There is a critical need for developing health services and training adequate health manpower to provide basic information and care for the health needs of the human and animal populations.

At the very least, it is worth considering what could be accomplished simply by supporting studies on the evidemiology of the animal parasitic diseases. It is an activity which could involve large numbers of untrained young people in an important scientific and educational enterprise. It would serve to develop a pool of talent from which the best could be selected for more advanced training in the health sciences and services.

\section{Behind the politics of influenza}

The idea of the United States government footing the bill to prevent the American population falling sick makes President Ford's request for funds to attempt a mass inoculation against flu something more than a medical issue in this election year. From Washington, Colin Norman gives the background

ON FEBRUARY 3, a young army recruit stationed at Fort Dix, New Jersey, came down with what seemed like a typical case of influenza. But the next day, while on a training march, he collapsed and died. His death sparked off an extraordinary series of events, culminating last week in the launching of the most extensive and controversial mass inoculation campaign ever undertaken in the United States.

The cause of the soldier's death turned out to be pneumonia, but a virus isolated from his respiratory tract immediately set alarm bells ringing in public health laboratories around the world. It was found to be a strain of influenza virus, commonly found in swine, which had not been involved in human infections for nearly 50 years. The virus, moreover, is very similar to the strain which caused the most serious flu pandemic in modern history, the 1918-19 outbreak which swept around the world killing some 20 million people.

Though only 12 other cases of swine flu were subsequently confirmed at Fort Dix, a massive monitoring effort on the base found antibodies to the flu strain in the blood of some 500 recruits, suggesting that they had been infected with swine flu during the outbreak. If the young recruit hadn't died, the outbreak would simply have been put down to the so-called Victoria/A strain which has been widespread in the United States this winter, but the isolation of the swine virus may have provided advance warning of a possible new flu pandemic next winter.

Because swine flu hasn't been abroad in the human population since the late $1930 \mathrm{~s}$, nobody under the age of 50 has any antibodies to guard against it. And it is likely that even those who do have antibodies are not protected against the disease. Thus, if the Fort Dix outbreak is not just a freak, isolated event, the virus could spread like wildfire through an unprotected population. $\mathrm{Dr}$ Edwin Kilbourne, a leading flu researcher at Mount Sinai School of Medicine in New York, said last week, for example, that "our thought is that probably the virus is seeded now in foci around the country, and when winter comes we will see it again".

For several weeks after the virus was isolated and identified, committees of scientists and government officials met at the US government's Center for Disease Control in Atlanta, Georgia, to decide what, if anything, should be done to counter the potential threat. Last week, they decided to recommend a massive inoculation effort against the virus, and President Ford appeared at a press conference, flanked by two of the most well known scientists in the United States-Jonas Salk and Albert Sabin-to announce that he is requesting $\$ 135$ million from Congress for a crash programme to inoculate every man, woman and child in the United States by the end of November. 